\title{
THE HANKEL TRANSFORMATION ON $M_{\mu}^{\prime}$ AND ITS REPRESENTATION
}

\author{
E. L. KOH AND C. K. LI
}

(Communicated by Palle E. T. Jorgensen)

ABstract. The Hankel transformation was extended by Zemanian to certain generalized functions of slow growth through a generalization of Parseval's equation as

$$
\left\langle h_{\mu} f, \varphi\right\rangle=\left\langle f, h_{\mu} \varphi\right\rangle
$$

where $\varphi, h_{\mu} \varphi \in H_{\mu}, f \in H_{\mu}^{\prime}$.

Later, Koh and Zemanian defined the generalized complex Hankel transformation on $J_{\mu}=\bigcup_{\nu=1}^{\infty} J_{a_{\nu}, \mu}$, where $J_{a_{\nu}, \mu}$ is the testing function space which contains the kernel function, $\sqrt{x y} J_{\mu}(x y)$. A transformation was defined directly as the application of a generalized function to the kernel function, i.e., for $f \in J_{\mu}^{\prime}$,

$$
\left(h_{\mu} f\right)(y)=\left\langle f(x), \sqrt{x y} J_{\mu}(x y)\right\rangle .
$$

In this paper, we extend definition (2) to a larger space of generalized functions. We first introduce the test function space $M_{a, \mu}$ which contains the kernel function and show that $H_{\mu} \subset M_{a, \mu} \subset J_{a, \mu}$. We then form the countable union space $M_{\mu}=\bigcup_{\nu=1}^{\infty} M_{a_{\nu}, \mu}$ whose dual $M_{\mu}^{\prime}$ has $J_{\mu}^{\prime}$ as a subspace. Our main result is an inversion theorem stated as follows.

Let $F(y)=\left(h_{\mu} f\right)(y)=\left\langle f(x), \sqrt{x y} J_{\mu}(x y)\right\rangle, f \in M_{\mu}^{\prime}$, where $y$ is restricted to the positive real axis. Let $\mu \geq-\frac{1}{2}$. Then, in the sense of convergence in $H_{\mu}^{\prime}$,

$$
f(x)=\lim _{r \rightarrow \infty} \int_{0}^{r} F(y) \sqrt{x} y J_{\mu}(x y) d y .
$$

This convergence gives a stronger result than the one obtained by Koh and Zemanian (1968).

Secondly, we prove that every generalized function belonging to $M_{a, \mu}^{\prime}$ can be represented by a finite sum of derivatives of measurable functions. This proof is analogous to the method employed in structure theorems for Schwartz distributions (Edwards, 1965), and similar to one by Koh (1970).

Received by the editors March 10, 1993.

1991 Mathematics Subject Classification. Primary 46F12, 46F10, 44A15.

Key words and phrases. Hankel transformation, generalized functions, countable union spaces, inversion theorem. 


\section{INTRODUCTION}

The conventional Hankel transformation is defined by

$$
F(y)=h_{\mu} f=\int_{0}^{\infty} f(x) \sqrt{x y} J_{\mu}(x y) d x,
$$

where $0<y<\infty, \mu$ is a real number, and $J_{\mu}$ is the Bessel function of the first kind and order $\mu$. In 1966, Zemanian (see [4]) constructed a testing function space $H_{\mu}$ in order to extend this transformation to certain generalized functions.

For each real number $\mu$, a function $\varphi(x)$ is in $H_{\mu}$ if and only if it is defined on $0<x<\infty$, it is complex-valued and smooth, and for each pair of nonnegative integers $m$ and $k$,

$$
\gamma_{m, k}^{\mu}(\varphi) \triangleq \sup _{0<x<\infty}\left|x^{m}\left(x^{-1} D\right)^{k}\left[x^{-\mu-1 / 2} \varphi(x)\right]\right|
$$

exists (i.e., is finite). $H_{\mu}$ is a linear space. Also, each $\gamma_{m, k}^{\mu}$ is a seminorm on $H_{\mu}$. The topology of $H_{\mu}$ is that generated by $\left\{\gamma_{m, k}^{\mu}\right\}_{m, k=0}^{\infty}$.

The Hankel transformation $h_{\mu}$ is an automorphism on $H_{\mu}$ whenever $\mu \geq$ $-\frac{1}{2}$. The generalized functions in the dual $H_{\mu}^{\prime}$ of $H_{\mu}$ act like distributions of slow growth as $x \rightarrow \infty$. Moreover, $H_{\mu}^{\prime}$ is the domain of the generalized Hankel transformation $h_{\mu}$, which is defined via (1). It follows that $h_{\mu}$ is an automorphism on $H_{\mu}^{\prime}$. This procedure is reminiscent of Schwartz's method of extending the Fourier transformation to distributions of slow growth.

In 1968, Koh and Zemanian [1] developed an alternative approach to the generalized Hankel transformation. For a real number $\mu$ and a positive real number $a$, they constructed a testing function space $J_{a, \mu}$ as follows.

Let $J_{a, \mu}$ be a testing function space containing all $\varphi(x)$ which are defined and smooth on $I=(0, \infty)$ and for which

$$
\begin{aligned}
\tau_{k}^{\mu, a}(\varphi) & =\sup _{x \in I}\left|e^{-a x} x^{-\mu-1 / 2}\left(x^{-\mu-1 / 2} D x^{2 x+1} D x^{-\mu-1 / 2}\right)^{k}(\varphi)\right| \\
& <\infty, \quad k=0,1,2, \ldots
\end{aligned}
$$

They assign to $J_{a, \mu}$ the topology generated by the countable multinorm $\left\{\tau_{k}^{\mu, a}\right\}_{k=0}^{\infty}$.

$J_{a, \mu}$ contains the kernel $\sqrt{x y} J_{\mu}(x y)$ as a function on $0<x<\infty$ for each fixed complex $y$ in the strip $\Omega=\{y:|\operatorname{Im} y|<a, y \neq 0$ or a negative number $\}$. The Hankel transformation $h_{\mu}$ is now defined on the dual space $J_{a, \mu}^{\prime}$ via (2).

It is proved in [1] that any generalized function that has a Hankel transform according to (2) will also have a Hankel transform according to (1), and the two transforms will agree.

The definition (2), while not as general as that of (1), is a more natural extension of the classical transformation because the kernel appears explicitly as a testing function. This leads to simpler manipulation for computational purposes. This could not be done in (1) because the kernel is not a member of $H_{\mu}$, whatever be the value of $y$.

In this paper, we define a new testing function space $M_{a, \mu}$ between $H_{\mu}$ and $J_{a, \mu}$, namely $H_{\mu} \subset M_{a, \mu} \subset J_{a, \mu}$, whereby $M_{a, \mu}$ still contains the kernel function. Since $J_{a, \mu}^{\prime} \subset M_{a, \mu}^{\prime}$, definition (2) is thus extended to a larger class of 
generalized functions. We obtain many properties of $M_{a, \mu}$ and the countable union $M_{\mu}=\bigcup_{i=1}^{\infty} M_{a_{i}, \mu}$. An inversion theorem and a representation for $M_{a, \mu}^{\prime}$ are our main results.

By a smooth function, we mean a function that possesses continuous ordinary derivatives of all orders at all points of its domain. The notation $\langle f, \varphi\rangle$ denotes the number assigned to a member $\varphi$ of some testing function space by a member $f$ of the dual space.

$D(I)$ denotes the space of smooth functions that have compact support on $I$. We equip $D(I)$ with the usual topology. Its dual $D^{\prime}(I)$ is the space of Schwartz distributions on $I$.

$E(I)$ denotes the space of smooth functions on $I$. Its dual $E^{\prime}(I)$ is the space of distributions with compact support on $I$.

The following theorem given in [4] will be used subsequently.

Theorem 1.1. If $f(x) \in L_{1}(0, \infty)$, if $f(x)$ is of bounded variation in a neighbourhood of the point $x=x_{0}>0$, if $\mu \geq-\frac{1}{2}$, and if $F(y)$ is defined as the conventional Hankel transformation, then

$$
\frac{1}{2}\left[f\left(x_{0}+0\right)+f\left(x_{0}-0\right)\right]=h_{\mu}^{-1} F=\int_{0}^{\infty} F(y) \sqrt{x_{0} y} J_{\mu}\left(x_{0} y\right) d y .
$$

Note that when $\mu \geq-\frac{1}{2}$, the conventional inverse Hankel transformation $h_{\mu}^{-1}$ is defined by precisely the same formula as is the direct Hankel transformation $h_{\mu}$; in symbols, $h_{\mu}=h_{\mu}^{-1}$.

\section{The TESTING FUNCTION SPACES $M_{a, \mu}$ AND $M_{\mu}$}

Let $a \in I$ and $\mu \in R$. We define $M_{a, \mu}$ as the space of testing functions $\varphi(x)$ which are defined and smooth on $0<x<\infty$, taking its value in $C$, and for which

$$
\gamma_{m, k}^{a, \mu}(\varphi)=\sup _{x \in I}\left|e^{-a x} x^{m}\left(x^{-1} D\right)^{k} x^{-\mu-1 / 2} \varphi(x)\right|<\infty, \quad m, k=0,1,2, \ldots
$$

We assign to $M_{a, \mu}$ the topology generated by the countable multinorm $\left\{\gamma_{m, k}^{a, \mu}\right\}$. $M_{a, \mu}$ is a Hausdorff space since $\gamma_{m, 0}^{a, \mu}$ is a norm.

The following properties will be inferred.

(i) Let $\mu \geq-\frac{1}{2}$. For a fixed complex number $y$ belonging to the strip $\Omega=\{y:|\operatorname{Im} y|<a, y \neq 0$ or a negative number $\}$,

$$
\frac{\partial^{m}}{\partial y^{m}}\left(\sqrt{x y} J_{\mu}(x y)\right) \in M_{a, \mu}
$$

Indeed, it is easily verified that (see [1])

$$
\frac{\partial^{m}}{\partial y^{m}}\left(\sqrt{x y} J_{\mu}(x y)\right)=\sum_{j=0}^{m} a_{j}(\mu) y^{j-m} x^{i} \sqrt{x y} J_{\mu-j}(x y),
$$

where the $a_{j}(\mu)$ are constants depending on $\mu$ only.

Considering

$$
\begin{aligned}
\left(x^{-1} D\right)^{k} x^{-\mu-1 / 2} x^{j} \sqrt{x y} J_{\mu-j}(x y) & =\sqrt{y}\left(x^{-1} D\right)^{k} x^{-(\mu-j)} J_{\mu-j}(x y) \\
& =(-1)^{k} y^{k+1 / 2} x^{-(\mu-j+k)} J_{\mu-j+k}(x y)
\end{aligned}
$$


and

$$
\begin{aligned}
(x y)^{-(\mu-j+k)} J_{\mu-j+k}(x y) & \sim \frac{1}{2^{\mu-j+k} \Gamma(\mu-j+k+1)} \text { as } x \rightarrow 0^{+} \\
& =O\left[(x y)^{-(\mu-j+k)-1 / 2} e^{x|\operatorname{Im} y|}\right] \text { as } x \rightarrow \infty,
\end{aligned}
$$

it follows that

$$
\gamma_{m, k}^{a, \mu}\left(x^{j} \sqrt{x y} J_{\mu-j}(x y)\right)<\infty
$$

Therefore

$$
\gamma_{m, k}^{a, \mu}\left[\frac{\partial^{m}}{\partial y^{m}}\left(\sqrt{x y} J_{\mu}(x y)\right)\right] \leq \sum_{j=1}^{m}\left|a_{j}(\mu)\right| \cdot|y|^{j-m} \gamma_{m, k}^{a, \mu}\left(x^{j} \sqrt{x y} J_{\mu-j}(x y)\right)<\infty
$$

for a fixed $y \in \Omega$.

(ii) The differential operator $N_{\mu} \triangleq x^{\mu+1 / 2} D x^{-\mu-1 / 2}$ is continuous from $M_{a, \mu}$ into $M_{a, \mu+1}$. Indeed

$$
\gamma_{m, k}^{a, \mu+1}\left(N_{\mu} \varphi\right)=\gamma_{m, k+1}^{a, \mu}(\varphi)
$$

Note: It is impossible for us to define $N_{\mu}^{-1}$ on $M_{a, \mu+1}$.

The differential operator $\bar{M}_{\mu} \triangleq x^{-\mu-1 / 2} D x^{\mu+1 / 2}$ is continuous from $M_{a, \mu+1}$ into $M_{a, \mu}$. Indeed,

$$
\gamma_{m, k}^{a, \mu}\left(\bar{M}_{\mu} \varphi\right) \leq 2|\mu+k+1| \gamma_{m, k}^{a, \mu+1}(\varphi)+\gamma_{m+2, k+1}^{a, \mu+1}(\varphi) .
$$

(iii) Multipliers in $M_{a, \mu}$. Define

$O=\left\{\theta(x) \in C_{I}^{\infty} \mid \exists C_{\nu} \in I\right.$ for each integer $\nu \geq 0, \exists$ integer $n_{\nu}$, such that

$$
\left.\left|\frac{\left(x^{-1} D\right)^{\nu} \theta(x)}{1+x^{n_{\nu}}}\right| \leq C_{\nu}\right\}
$$

For arbitrary $\theta \in O$ and $\varphi \in M_{a, \mu}$, we have

$$
\left(x^{-1} D\right)^{k} x^{-\mu-1 / 2} \theta \varphi=\sum_{\nu=0}^{k}\left(\begin{array}{l}
k \\
\nu
\end{array}\right) \frac{\left(x^{-1} D\right)^{\nu} \theta}{1+x^{n_{\nu}}}\left(1+x^{n \nu}\right)\left(x^{-1} D\right)^{k-\nu} x^{-\mu-1 / 2} \varphi
$$

so that

$$
\gamma_{m, k}^{a, \mu}(\theta \varphi) \leq \sum_{\nu=0}^{k}\left(\begin{array}{l}
k \\
\nu
\end{array}\right) C_{\nu}\left[\gamma_{m, k-\nu}^{a, \mu}(\varphi)+\gamma_{m+n_{\nu}, k-\nu}^{a, \mu}(\varphi)\right] .
$$

(iv) $M_{a, \mu}$ is complete. The proof is very similar to Lemma 5.2.2 on page 131 in [4].

(v) $H_{\mu} \subset M_{a, \mu}$ for all $\mu \in R, a \in I$. And the topology of $H_{\mu}$ is stronger than that induced on it by $M_{a, \mu}$. Indeed, $e^{-a x} \leq 1$ on $(0, \infty)$ and $\gamma_{m, k}^{a, \mu}(\varphi) \leq$ $\gamma_{m, k}^{\mu}(\varphi)$.

Our space $M_{a, \mu}$ is a subspace of $J_{a, \mu}$ and has a stronger topology than that induced on it by $J_{a, \mu}$.

To see $M_{a, \mu} \subset J_{a, \mu}$, we note that

$$
x^{-\mu-1 / 2} S_{\mu}^{k} \varphi=\sum_{j=0}^{k} b_{j} x^{2 j}\left(x^{-1} D\right)^{k+j} x^{-\mu-1 / 2} \varphi
$$


where $S_{\mu}=x^{-\mu-1 / 2} D x^{2 \mu+1} D x^{-\mu-1 / 2}$, and the constants $b_{j}$ depend on $\mu$ only. It follows that

$$
\tau_{k}^{a, \mu}(\varphi) \leq \sum_{j=0}^{k}\left|b_{j}\right| \gamma_{2 j, k+j}^{a, \mu}(\varphi)
$$

for any $\varphi \in M_{a, \mu}$. This implies our assertion.

Note that $H_{\mu}$ is a proper subset of $M_{a, \mu}$. Indeed, $\sqrt{x y} J_{\mu}(x y) \in M_{a, \mu}$ as was already shown in (i), but $\sqrt{x y} J_{\mu}(x y) \notin H_{\mu}$ since it is not of rapid descent. Also, $M_{a, \mu}$ is a proper subset of $J_{a, \mu}$. In fact, we define

$$
\varphi_{1}(x)= \begin{cases}e^{a x}, & x \geq 1, \\ \text { smooth } & 1 / 2<x<1, \\ 0, & 0<x \leq 1 / 2 .\end{cases}
$$

Let $\varphi=x^{\mu+1 / 2} \varphi_{1}(x)$, then $\varphi(x) \in J_{a, \mu}$. By equation (3) we have

$$
e^{-a x} x^{-\mu-1 / 2} S_{\mu}^{k} \varphi=\sum_{j=0}^{k} b_{j} e^{-a x} x^{2 j}\left(x^{-1} D\right)^{k+j} x^{-\mu-1 / 2} \varphi .
$$

When $x \geq 1$

$$
e^{-a x} x^{-\mu-1 / 2} S_{\mu}^{k} \varphi=\sum_{j=0}^{k} b_{j} e^{-a x} x^{2 j}\left(x^{-1} D\right)^{k+j} e^{a x} .
$$

It is easily verified that

$$
\left(x^{-1} D\right)^{k+j} e^{a x}=C(k, j) x^{-(2 k+2 j)+1} a e^{a x}+\cdots+a^{k+j} x^{-k-j} e^{a x},
$$

where $C(k, j)$ is a constant depending on $k, j$.

It follows that $\tau_{k}^{a, \mu}(\varphi)<\infty$, but $\gamma_{1,0}^{a, \mu}(\varphi) \geq \sup _{x \in[1, \infty)}\left|e^{-a x} x e^{a x}\right|=\infty$.

(vi) If $a>b>0$, then $M_{b, \mu} \subset M_{a, \mu}$ and the topology of $M_{b, \mu}$ is stronger than that induced on it by $M_{a, \mu}$. This follows immediately from the inequality

$$
\gamma_{m, k}^{a, \mu}(\varphi) \leq \gamma_{m, k}^{b, \mu}(\varphi) \text { for } \varphi \in M_{b, \mu} .
$$

(vii) $D(I) \subset M_{a, \mu}$ and the topology of $D(I)$ is stronger than that induced on it by $M_{a, \mu}$. By the way, we point out that $D(I)$ is not dense in $M_{a, \mu}$.

(viii) For every choice of $\mu$ and $a, M_{a, \mu} \subset E(I)$. Moreover, it is dense in $E(I)$ because $D(I)$ is dense in $E(I)$.

(ix) For each $f \in M_{a, \mu}^{\prime}$, there exist a pair of nonnegative integers $m_{0}, k_{0}$ and a positive constant $C$ such that for $\varphi \in M_{a, \mu}$

$$
|\langle f, \varphi\rangle| \leq C \max _{\substack{0 \leq k \leq k_{0} \\ 0 \leq m \leq m_{0}}} \gamma_{m, k}^{a, \mu}(\varphi) .
$$

We turn now to the definition of a certain countable-union space $M_{\sigma, \mu}$ (for short $M_{\mu}$ ) that arises from the $M_{a, \mu}$ spaces. Our subsequent discussion takes on a simpler form when the space $M_{\mu}$ is used in place of the $M_{a, \mu}$ spaces. Let $\left\{a_{\nu}\right\}_{\nu=1}^{\infty}$ be a monotonically increasing sequence of pcsitive numbers tending to $\sigma$. Here $\sigma=\infty$ is allowed. By virtue of note (vi), $\left\{M_{a_{\nu}, \mu}\right\}_{\nu=1}^{\infty}$ is a sequence of Fréchet spaces such that $M_{a_{1}, \mu} \subset M_{a_{2}, \mu} \subset \cdots$, and such that the topology of $M_{a_{\nu}, \mu}$ is stronger than that induced on it by $M_{a_{\nu+1}, \mu}$. Let $M_{\sigma, \mu}\left(=M_{\mu}\right)=$ 
$\bigcup_{\nu=1}^{\infty} M_{a_{\nu}, \mu}$ denote the countable-union space generated by the above sequence of spaces. Thus, a sequence $\left\{\varphi_{n}\right\}_{n=1}^{\infty}$ converges in $M_{\mu}$ to $\varphi$ iff for some fixed $a_{\nu}, \varphi_{n}, \varphi \in M_{a_{\nu}, \mu}$ and $\varphi_{n} \rightarrow \varphi$ in $M_{a_{\nu}, \mu}$.

We assign to $M_{\mu}^{\prime}$ the usual weak convergence concept. Accordingly, a sequence $\left\{f_{\nu}\right\}_{\nu=1}^{\infty}$ converges in $M_{\mu}^{\prime}$ if there exists an $f \in M_{\mu}^{\prime}$ such that, for every $\varphi \in M_{\mu}$

$$
\left|\left\langle f_{\nu}, \varphi\right\rangle-\langle f, \varphi\rangle\right| \rightarrow 0 \text { as } \nu \rightarrow \infty .
$$

The following lemmas are immediate.

Lemma 2.1. For any fixed complex number y belonging to $\Omega=\{y:|\operatorname{Im} y|<\sigma$, $y \neq 0$ or a negative number $\}$

$$
\frac{\partial^{m}}{\partial y^{m}}\left(\sqrt{x y} J_{\mu}(x y)\right) \in M_{\mu}, \quad m=0,1,2, \ldots
$$

Lemma 2.2. For every choice of $\sigma>0, H_{\mu} \subset M_{\mu}$ and convergence in $H_{\mu}$ implies convergence in $M_{\mu}$.

\section{THE GENERALIZEd HANKel TRANSFORMATION ON $M_{\mu}^{\prime}$}

Let $\mu \geq-\frac{1}{2}$. In view of (vi), to every $f \in M_{a, \mu}^{\prime}$ there exists a unique real number $\sigma_{f}$ possibly $\sigma_{f}=\infty$ such that $f \in M_{b, \mu}^{\prime}$ if $b<\sigma_{f}$ and $f \notin M_{b, \mu}^{\prime}$ if $b>\sigma_{f}$. Therefore, $f \in M_{\sigma_{f}, \mu}^{\prime}$. We define the $\mu$ th order Hankel transform $h_{\mu} f$ as the application of $f$ to the kernel $\sqrt{x y} J_{\mu}(x y)$; i.e.,

$$
F(y)=\left(h_{\mu} f\right)(y)=\left\langle f(x), \sqrt{x y} J_{\mu}(x y)\right\rangle,
$$

where $y \in \Omega_{f}=\left\{y:|\operatorname{Im} y|<\sigma_{f}, y \neq 0\right.$ or a negative number $\}$. The strip $\Omega_{f}$ will be called the region of definition for $F(y)$.

The following results given in [5] will be needed.

Lemma 3.1. Let $a$ be a fixed positive number. For all $y$ in the strip $\Omega=$ $\{y:|\operatorname{Im} y|<a, y \neq 0$ or a negative number $\}$, for $0<x<\infty$, and for $\mu \geq-\frac{1}{2}$,

$$
\left|e^{-a x}(x y)^{-\mu} x^{m} J_{\mu}(x y)\right| \leq A_{\mu},
$$

where $A_{\mu}$ is a constant with respect to $x$ and $y$.

Lemma 3.2 (Boundedness of $F(y)) . F(y)$ is bounded on any cut strip $\{y:|\operatorname{Im} y|$ $\leq a_{1}<a<\sigma_{f}, y \neq 0$ or a negative number $\}$ according to

$$
|F(y)| \leq|y|^{\mu+1 / 2} P_{a}(|y|),
$$

where $P_{a}(|y|)$ is a polynomial depending only on $a$.

Lemma 3.3 (Analyticity of $F(y)$ ). $F(y)$ is an analytic function in $\Omega=\{y$ : $|\operatorname{Im} y| \leq a<\sigma_{f}, y \neq 0$ or a negative number $\}$ and

$$
D_{y} F(y)=\left\langle f(x), \frac{\partial}{\partial y} \sqrt{x y} J_{\mu}(x y)\right\rangle .
$$

Theorem 3.1. Let $f \in M_{\mu}^{\prime}, \varphi \in H_{\mu}$, and $\mu \geq-\frac{1}{2}$. Then

$$
\left\langle\left\langle f(x), \sqrt{x y} J_{\mu}(x y)\right\rangle, \varphi(y)\right\rangle=\left\langle f(x), \int_{0}^{\infty} \sqrt{x y} J_{\mu}(x y) \varphi(y) d y\right\rangle, \quad y \in I .
$$


Proof. Since $\left\langle f(x), \sqrt{x y} J_{\mu}(x y)\right\rangle$ is of slow growth as $y \rightarrow \infty$ by Lemma 3.2, and is Lebesgue integrable on $0<y<Y$ for $Y \in(0, \infty)$ by Lemma 3.3, we can write

$$
\left\langle\left\langle f(x), \sqrt{x y} J_{\mu}(x y)\right\rangle, \varphi(y)\right\rangle=\int_{0}^{\infty}\left\langle f(x), \sqrt{x y}, J_{\mu}(x y)\right\rangle \varphi(y) d y
$$

since $\varphi \in H_{\mu}$.

Our theorem will be proven when we show that

$$
\int_{0}^{\infty}\left\langle f(x), \sqrt{x y} J_{\mu}(x y)\right\rangle \varphi(y) d y=\left\langle f(x), \int_{0}^{\infty} \varphi(y) \sqrt{x y} J_{\mu}(x y) d y\right\rangle .
$$

Consider the Riemann sum

$$
\frac{Y}{m} \sum_{\nu=1}^{m} \varphi\left(\nu \frac{Y}{m}\right)\left\langle f(x), \sqrt{x \nu \frac{Y}{m}} J_{\mu}\left(x \nu \frac{Y}{m}\right)\right\rangle .
$$

This sum converges to the integral $\int_{0}^{Y} \varphi(y)\left\langle f(x), \sqrt{x y} J_{\mu}(x y)\right\rangle d y$ for the integrand is continuous on $0<y<Y$. Moreover, since $\varphi(y)$ is of rapid descent while $\left\langle f(x), \sqrt{x y} J_{\mu}(x y)\right\rangle$ is bounded by a polynomial in $|y|$, the last integral converges to the right-hand side of (4) as $Y \rightarrow \infty$.

On the other hand, we are able to write (5) as

$$
\left\langle f(x), \frac{Y}{m} \sum_{\nu=1}^{m} \varphi\left(\nu \frac{Y}{m}\right) \sqrt{x \nu \frac{Y}{m}} J_{\mu}\left(x \nu \frac{Y}{m}\right)\right\rangle
$$

and show that, as $m$ and then $Y \rightarrow \infty$, (6) converges to

$$
\left\langle f(x), \int_{0}^{\infty} \varphi(y) \sqrt{x y} J_{\mu}(x y) d y\right\rangle .
$$

Indeed, by taking the operator $\left(x^{-1} D\right)^{k} x^{-\mu-1 / 2}$ under the integral and the summation signs, we have

$$
\begin{aligned}
\gamma_{m, k}^{a, \mu}\{ & \left.\left.\int_{0}^{Y} \varphi(y) \sqrt{x y} J_{\mu}(x y) d y-\frac{Y}{m} \sum_{\nu=1}^{m} \varphi\left(\nu \frac{Y}{m}\right) \sqrt{x \nu \frac{Y}{m}} J_{\mu}\left(x \nu \frac{Y}{m}\right)\right\}\right\} \\
= & \sup _{x \in I} \mid e^{-a x} x^{m}\left\{\int_{0}^{Y} \varphi(y)(-1)^{k} y^{k+1 / 2} x^{-(\mu+k)} J_{\mu+k}(x y) d y\right. \\
& \left.-\frac{Y}{m} \sum_{\nu=1}^{m} \varphi\left(\nu \frac{Y}{m}\right)(-1)^{k}\left(\nu \frac{Y}{m}\right)^{\mu+2 k+1 / 2}\left(x \nu \frac{Y}{m}\right)^{-(\mu+k)} J_{\mu+k}\left(x \nu \frac{Y}{m}\right)\right\} \mid .
\end{aligned}
$$

Because of the factor $e^{-a x} x^{m}$ and the boundedness of $(x y)^{-(\mu+k)} J_{\mu+k}(x y)$ on $0<x y<\infty$, given an $\varepsilon>0$, there exists an $X$ such that for all $x>X$, the quantity under the supremum sign is less than $\varepsilon$ for every $m$. Now, on $0<x<X, 0<y<Y$, the expression $\varphi(y) y^{\mu+2 k+1 / 2}(x y)^{-\mu+k} J_{\mu+k}(x y)$ is uniformly continuous, hence the Riemann sum on the right-hand side of (7) converges to the integral uniformly on $0<x<X$ as $m \rightarrow \infty$. This, (6) converges to $\left\langle f(x), \int_{0}^{Y} \varphi(y) \sqrt{x y} J_{\mu}(x y) d y\right\rangle$ as $m \rightarrow \infty$. 
Finally, we show that $\int_{Y}^{\infty} \varphi(y) \sqrt{x} y J_{\mu}(x y) d y \rightarrow 0$ as $Y \rightarrow \infty$ in $M_{\mu}$. This is because of the following inequalities:

$$
\begin{aligned}
\mid e^{-a x} & x^{m}\left(x^{-1} D\right)^{k} x^{-\mu-1 / 2} \int_{Y}^{\infty} \varphi(y) \sqrt{x y} J_{\mu}(x y) d y \mid \\
= & \left|\int_{Y}^{\infty} \varphi(y) e^{-a x} x^{m}(-1)^{k} y^{\mu+2 k+1 / 2}(x y)^{-(\mu+k)} J_{\mu+k}(x y) d y\right| \\
\leq & A_{\mu} \int_{Y}^{\infty}\left|\varphi(y) y^{\mu+2 k+1 / 2}\right| d y \rightarrow 0 .
\end{aligned}
$$

The last inequality is due to Lemma 3.1.

Inversion and uniqueness. We now state an inversion theorem for our generalized Hankel transformation.

Theorem 3.2. Let $F(y)=\left\langle f(x), \sqrt{x y} J_{\mu}(x y)\right\rangle, f \in M_{\mu}^{\prime}, y \in I$. Let $\mu \geq-\frac{1}{2}$. Then, in the sense of convergence in $H_{\mu}^{\prime}$,

$$
f(x)=\lim _{r \rightarrow \infty} \int_{0}^{r} F(y) \sqrt{x y} J_{\mu}(x y) d y .
$$

Proof. Let $\varphi(x) \in H_{\mu}$, we wish to show that

$$
\left\langle\int_{0}^{r} F(y) \sqrt{x y} J_{\mu}(x y) d y, \varphi(x)\right\rangle
$$

tends to $\langle f(x), \varphi(x)\rangle$ as $r \rightarrow \infty$. Since $F(y)$ is smooth and $\sqrt{x y} J_{\mu}(x y)$ is bounded on $0<x y<\infty$, it follows that $\int_{0}^{r} F(y) \sqrt{x y} J_{\mu}(x y) d y$ is continuous and bounded with respect to $x$. Hence we have

$$
\left\langle\int_{0}^{r} F(y) \sqrt{x y} J_{\mu}(x y) d y, \varphi(x)\right\rangle=\int_{0}^{\infty} \int_{0}^{r} F(y) \sqrt{x y} J_{\mu}(x y) d y \varphi(x) d x .
$$

By Fubini's theorem we can change the order of integration and obtain

$$
\begin{aligned}
\int_{0}^{\infty} & \varphi(x) \int_{0}^{r} F(y) \sqrt{x y} J_{\mu}(x y) d y d x \\
& =\int_{0}^{r}\left\langle f(x), \sqrt{x y} J_{\mu}(x y)\right\rangle \int_{0}^{\infty} \varphi(x) \sqrt{x y} J_{\mu}(x y) d x d y .
\end{aligned}
$$

Set $\Phi(y)=\int_{0}^{\infty} \varphi(x) \sqrt{x y} J_{\mu}(x y) d x$. Then $\Phi(y) \in H_{\mu}$ since $\mu \geq-\frac{1}{2}$.

By Theorem 3.1, the right-hand side of (8) can be written as

$$
\left\langle f(x), \int_{0}^{r} \sqrt{x y} J_{\mu}(x y) \int_{0}^{\infty} \varphi(x) \sqrt{x y} J_{\mu}(x y) d x d y\right\rangle .
$$

Now, we wish to show

$$
L_{r}(x)=\int_{0}^{r} \sqrt{t y} J_{\mu}(t y) \int_{0}^{\infty} \varphi(x) \sqrt{x y} J_{\mu}(x y) d x d y
$$

converges in $M_{\mu}$ to $\varphi(t)$ as $r \rightarrow \infty$.

By the last part of the proof of Theorem 3.1, we get

$$
\lim _{r \rightarrow \infty} \int_{0}^{r} \sqrt{x y} J_{\mu}(x y) \Phi(y) d y=\int_{0}^{\infty} \sqrt{x y} J_{\mu}(x y) \Phi(y) d y=\varphi(x) .
$$

The last equality is due to Theorem 1.1.

As a result of the inversion theorem, we have the following theorem. 
Theorem 3.3. Let $F(y)=h_{\mu} f=G(y)=h_{\mu} g$ for $y \in I$, then $f=g$ in the sense of equality in $H_{\mu}^{\prime}$.

Now, we come to prove a characterization theorem for the generalized function in $M_{a, \mu}^{\prime}$.

Theorem 3.4. A functional $f$ is in $M_{a, \mu}^{\prime}$ if and only if there exist bounded measurable functions $g_{m, k}(x)$ defined on $I$, for $m=0,1,2, \ldots, m_{0}$ and $k=0,1,2, \ldots, k_{0}$, where $m_{0}$ and $k_{0}$ are nonnegative integers depending on $f$, and such that

$$
\langle f, \varphi\rangle=\left\langle\sum_{m=0, k=0}^{m_{0}, k_{0}} x^{-\mu-1 / 2}\left(-D \frac{1}{x}\right)^{k}\left\{e^{-a x} x^{m}(-D) g_{m, k}(x)\right\}, \varphi(x)\right\rangle
$$

for every $\varphi \in M_{a, \mu}$.

Proof. Let $f \in M_{a, \mu}^{\prime}$. By using the property (ix), there exist a pair of nonnegative integers $m_{0}, k_{0}$, and a positive constant $C$ such that for $\varphi \in M_{a, \mu}$

$$
|\langle f, \varphi\rangle| \leq C \max _{\substack{0 \leq k \leq k_{0} \\ 0 \leq m \leq m_{0}}} \gamma_{m, k}^{a, \mu}(\varphi)=C \max _{\substack{0 \leq k \leq k_{0} \\ 0 \leq m \leq m_{0}}} \sup _{x \in I}\left|e^{-a x} x^{m}\left(x^{-1} D\right)^{k} x^{-\mu-1 / 2} \varphi\right| .
$$

Since $\varphi \in M_{a, \mu}$, there exists $C_{m, k}>0$ such that

$$
\sup _{x \in I}\left|e^{-a x} x^{m+2}\left(x^{-1} D\right)^{k} x^{-\mu-1 / 2} \varphi\right| \leq C_{m, k} \text { for fixed } a \text { and } \mu .
$$

It follows that

$$
\lim _{x \rightarrow \infty} e^{-a x} x^{m}\left(x^{-1} D\right)^{k} x^{-\mu-1 / 2} \varphi=0
$$

hence

$$
e^{-a x} x^{m}\left(x^{-1} D\right)^{k} x^{-\mu-1 / 2} \varphi=\int_{\infty}^{x} D_{t}\left\{e^{-a t} t^{m}\left(t^{-1} D\right)^{k} t^{-\mu-1 / 2} \varphi(t)\right\} d t
$$

From

$$
\begin{aligned}
& D_{t}\left\{e^{-a t} t^{m}\left(t^{-1} D\right)^{k} t^{-\mu-1 / 2} \varphi(t)\right\} \\
& \quad=D_{t}\left\{e^{-a t} t^{m}\right\}\left(t^{-1} D\right)^{k} t^{-\mu-1 / 2} \varphi(t)+e^{-a t} t^{m+1}\left(t^{-1} D\right)^{k+1} t^{-\mu-1 / 2} \varphi(t)
\end{aligned}
$$

and the fact that $\varphi \in M_{a, \mu}$, it follows that

$$
\begin{aligned}
\int_{0}^{\infty} & \left|D_{t}\left\{e^{-a t} t^{m}\left(t^{-1} D\right)^{k} t^{-\mu-1 / 2} \varphi(t)\right\}\right| d t \\
& =\left\|D_{t}\left\{e^{a t} t^{m}\left(t^{-1} D\right)^{k} t^{-\mu-1 / 2} \varphi(t)\right\}\right\|_{L_{1}(0, \infty)}
\end{aligned}
$$

is finite, where $\|\cdot\|_{L_{1}(0, \infty)}$ denotes the norm on the space $L_{1}(0, \infty)$. Then we have

$$
|\langle f, \varphi\rangle| \leq C \max _{\substack{0 \leq k \leq k_{0} \\ 0 \leq m \leq m_{0}}}|| D_{t}\left\{e^{-a t} t^{m}\left(t^{-1} D\right)^{k} t^{-\mu-1 / 2} \varphi(t)\right\}||_{L_{1}(0, \infty)} .
$$

Define an injective map $F_{1}: M_{a, \mu} \rightarrow F_{1} M_{a, \mu}$ by

$$
\begin{aligned}
\varphi \rightarrow\left(D_{t}\left\{e^{-a t} t^{m}\left(t^{-1} D\right)^{k} t^{-\mu-1 / 2} \varphi(t)\right\}\right), \quad & m=0,1, \ldots, m_{0}, \\
& k=0,1, \ldots, k_{0} .
\end{aligned}
$$


$F_{1} M_{a, \mu}$ is endowed with the topology induced on it by the product space $A_{m_{0}, k_{0}}=\left(L_{1}(0, \infty)\right)^{\left(m_{0}+1\right)\left(k_{0}+1\right)}$.

Define $F_{2}: F_{1} M_{a, \mu} \rightarrow C$ by $F_{1} \varphi \rightarrow\langle f, \varphi\rangle$. By virtue of $(10), F_{2}$ is a continuous linear mapping.

By applying the Hahn-Banach theorem, $F_{2}$ can be extended to $A_{m_{0}, k_{0}}$. Therefore, since $A_{m_{0}, k_{0}}^{\prime}$ is isomorphic to $\left(L_{\infty}(0, \infty)\right)^{\left(k_{0}+1\right)\left(m_{0}+1\right)}$ (see Treves [6]), there exist $\left(k_{0}+1\right)\left(m_{0}+1\right)$ bounded measurable functions $g_{m, k}(x)(m=$ $\left.0,1,2, \ldots, m_{0}, k=0,1,2, \ldots, k_{0}\right)$, such that

$$
\begin{aligned}
F_{2}\left(F_{1} \varphi\right)=\langle f, \varphi\rangle & =\sum_{m=0, k=0}^{m_{0}, k_{0}}\left\langle g_{m, k}(x), D_{x}\left\{e^{-a x} x^{m}\left(x^{-1} D\right)^{k} x^{-\mu-1 / 2} \varphi\right\}\right\rangle \\
& =\left\langle\sum_{m=0, k=0}^{m_{0}, k_{0}} x^{-\mu-1 / 2}\left(-D \frac{1}{x}\right)^{k}\left\{x^{m} e^{-a x}(-D) g_{m, k}(x)\right\}, \varphi\right\rangle .
\end{aligned}
$$

On the other hand, we assume $f$ is defined by (8). Obviously $f$ is linear. Let $\varphi_{n} \rightarrow 0$ in $M_{a, \mu}$, then $D_{x}\left\{e^{-a x} x^{m}\left(x^{-1} D\right)^{k} x^{-\mu-1 / 2} \varphi_{n}\right\}$ converges to 0 in $L_{1}(0, \infty)$. This completes the proof.

\section{REFERENCES}

1. E. L. Koh and A. H. Zemanian, The complex Hankel and I-transformations of generalized functions, SIAM J. Appl. Math. 16 (1968), 945-957.

2. E. L. Koh, A representation of Hankel transformable generalized functions, SIAM J. Math. Anal. 1 (1970), 33-36.

3. R. E. Edwards, Functional analysis, Holt, Rinehart, and Winston, New York, 1965.

4. A. H. Zemanian, Generalized integral transformations, Interscience, New York, 1968.

5. E. L. Koh and C. K. Li, The complex Hankel transformation on $M_{\mu}^{\prime}$, Congr. Numer. 87 (1992), 145-151.

6. F. Treves, Topological vector spaces distributions and kernels, Academic Press, New York, 1967.

Department of Mathematics and Statistics, University of Regina, Regina, SaskatcheWAN, CANADA S4S 0A2

E-mail address: elkoh@max.cc.uregina.ca

E-mail address: lichen@meena.cc.uregina.ca 